\title{
Chaos and cancers. Theories concerning carcinogenesis
}

\author{
Wojciech Kwasniewski ${ }^{1}$, Aleksandra Stupak ${ }^{2}$, Jan Kotarski ${ }^{1}$, Anna Gozdzicak-Jozefiak ${ }^{3}$ \\ ${ }^{1}$ Department of Gynecologic Oncology and Gynecology of the Medical University of Lublin, Poland \\ ${ }^{2}$ Chair and Department of Obstetrics and Pathology of Pregnancy, Medical University of Lublin, Poland \\ ${ }^{3}$ Department of Molecular Virology, Institute of Experimental Biology, Adam Mickiewicz University in Poznan, Poland
}

\begin{abstract}
One of the most intriguing problems in biomedical sciences is the theory explaining cancer formation. It is known that cancer is the result of many molecular processes, the presence of oncogenic factors and the loss of apoptosis of affected cells. We currently have hypotheses based on carcinogenesis because of a single cell gene mutation, i.e. somatic mutation theory (SMT), or disorders in tissue architecture and intercellular communication called (TOFT) Tissue Organization Field Theory. An attempt to combine these separate and compatible cause and effect pathways into one unified theory of cancer transformation is the theory of chaotic adaptation. The new interpretative model is the systemic-evolution theory of cancer (SETOC) which postulates disintegration between the symbiosis of "energy" and "information" in normal cells. There are also epidemiological studies confirming that some types of cancer arise from viral infection. So, let us ask the question, can one hypothesis explain all the features of cancer?
\end{abstract}

Key words: carcinogenesis; somatic mutation theory; tissue organization field theory; chaotic adaptation; systemic-evolution theory of cancer; genome; chaos; information

Ginekologia Polska 2021; 92, 4: 318-321

\section{INTRODUCTION}

Despite the spectacular contribution of molecular biology techniques in the field of carcinogenesis, the cancer mortality statistics do not show much difference. In the community of researchers of the process of neoplastic transformation, there is a debate regarding the insufficient methods of explaining this phenomenon. The purpose of our work is to present arguments, hypotheses and methodological assumptions describing the most current achievements in the context of the carcinogenesis dispute.

\section{THE THEORIES AND EVALUATION OF THE IDES}

For at least 30 years, the dominant theory of cancerogenesis was somatic mutation theory (SMT) [1]. It assumed that cancer is a clonal, cellular disorder, and its formation is a multistage process. It is characterized by the accumulation of changes in the genetic material in the cell - as a result of biological genotoxic mechanisms and epigenetic changes. The consequence of these changes is the accumulation of DNA mutations in tumor suppressor genes, microRNA genes, DNA repair genes or genes involved in cell cycle control and cell proliferation and apoptosis. In turn, epigenetic factors change the degree of DNA methylation and / or the conformation of chromatin, which is manifested by a change in the transcriptional activity of genes. Other factors important in the carcinogenesis process are also hereditary factors, immunology, hormonal status and external factors: chemicals, lifestyle, diet, cigarette smoke, sun exposure, radiation or viral, bacterial and other infections.

However, it should be emphasized that most cancers are histopathologically diverse and contain cytologically different clones resulting from genetic transformation from one transformed cell. It also seems that mutational changes are generally insufficient to cause cancer, because only a small proportion of cancers, in about $5 \%$, arise as a result of mutations [2]. The theory of somatic mutations has never been able to explain how non-mutagenic factors are responsible for carcinogenesis. The somatic mutation model also ignores the fact that in every known case of cancer, not only individual genes can contain mutations, but also entire chromosomes that carry thousands of genes, can be duplicated, damaged, or structurally incorrect. Numerous experimental data suggest that this chaos at the chromosomal level is not

\footnotetext{
Corresponding author:

Wojciech Kwasniewski

Department of Gynecologic Oncology and Gynecology of the Medical University of Lublin, Poland

e-mail: wojciech.kwasniewski@umlub.pl
}

This article is available in open access under Creative Common Attribution-Non-Commercial-No Derivatives 4.0 International (CC BY-NC-ND 4.0) license, allowing to download articles and share them with others as long as they credit the authors and the publisher, but without permission to change them in any way or use them commercially. 
only a side effect of carcinogenesis, but a direct cause and driving force of cancerous changes. Chromosomal mutations, which massively disturb the balance of thousands of genes, are sufficient to cause cellular instability [3]. Genetic instability may affect the enzymes which replicate DNA, enzymes which repair DNA, protein which affect chromosomal stability (histones, kinetochore proteins, spindle proteins) and proteins which control apoptosis and cell cycle regulation in response to DNA damage (p53 and pRb). The formation of micronuclei is also associated with chromosomal instability. Various molecular mechanisms are responsible for its formation, including double-stranded DNA breaks, impaired DNA repair response, improper DNA replication, DNA adduct-forming chemical or interference with mitosis [4]. Therefore, micronucleus formation usually acts as an index of genotoxic effects and chromosomal instability (both inherited and induced).

Therefore, carcinogens appear to act as "aneuploidies" rather than as mutagens. This fact explains why cancer cells, even within the same tumor, may have different combinations and changes in their chromosomes, making each cell a kind of new species for themselves, and their instability allows cancer cells to evolve new traits (phenotypes). The unusual variability of cancer cells and the huge variety of their phenotypes are the main reasons why tumors remain an unsolvable problem, both from a scientific and therapeutic point of view. The challenge, therefore, is to formulate a theory explaining how one normal cell, out of billions making up the human body, becomes chromosomally and phenotypically altered to cause a deadly cancer. Since each of the features of carcinogenesis that cannot be explained by a theory of mutation is associated with chromosomal changes, a chromosomal theory of cancer has been proposed that considers this inherent instability [5]. The SMT theory and the chromosomal theory are complemented by the theory called TOFT Tissue Organization Field Theory, which suggests that the origin and subsequent features of the cancer result from disorders in the microenvironment of the cell in the tissue in which the tumor is formed, lives and grows [6]. Molecular dialogue in this zone and cellular communication involves various host cells such as: endothelial cells, pericytes, immune cells, inflammatory cells, fibroblasts, soluble factors and structural components such as extracellular matrix, various proteins such as integrins, metalloproteinases, various factors growth and their receptors, as well as miRNA, oxygen, nutrient and other various chemical mediators [7]. Thus, the environment within the tumor and around the tumor becomes crucial for its growth, survival and impact on the host. According to the TOFT theory, tumorigenesis processes result from deregulation of interactions between cells and the microenvironment and disruption of cellular communication needed to main- tain normal tissue structure, and DNA mutations are not primarily a necessary carcinogen [6]. During the neoplastic process, significant structural and functional changes occur at the border between cancer cells and neighboring host cells. However, the cancer is still localized in single cells. Therefore, in TOFT DNA mutations are the result and not the cause of disorders at the tissue level.

Nevertheless, no theory can explain all the features of cancer. In other words, different types of cancer are characterized by uncontrolled cell proliferation, genomic instability, DNA damage, or significant reprogramming of cellular energy metabolism. All cancer cells are insensitive to signals that inhibit proliferation, allow cancer cells to avoid immune destruction, thereby acquiring neoangiogenesis capabilities and activating invasion and metastasis [8].

Bedessem and Ruphy suggest that TOFT and SMT describe two separate and compatible cause and effect pathways and that these two theories are not contradictory but converge and complement each other in one unified theory of carcinogenesis - chaotic adaptation theory (CAT) [9]. The essence of this theory is the hypothesis that cancer arises from the adaptation of stem cells. The main pillars of the theory are chaos „,adaptation „, and" information". According to CAT theory, the development of cancer is strictly dependent on the microenvironment. Two researchers Tomasetti and Vogelstein have shown that spontaneous mutations occurring during the division of stem cells can lead to the formation of neoplastic changes in some tissues, and the risk of developing cancerogenic changes is correlated with the total number of normal self-renewing cells that maintain tissue in homeostasis [10].

Normal somatic stem cells (SSCs) are described as immature cells that have a dual ability to self-renew and differentiate. Their activity can be explained by natural selection acting based on SSC decisions in response to signals from other SSCs in the local microenvironment and from more diverse cells in the rest of the body [11]. On the other hand, SSCs have remarkable regulatory flexibility that allows them to operate in various external conditions. This ability to adapt to environmental changes is important for all organisms to maintain cellular functions [12]. SSC function is controlled by coordinated activation and / or inhibition of thousands of genes in a particular environment.

According to the hypothesis of chaotic adaptation theory, somatic stem cells (SSCs) receive information from daughter cells, which is transmitted to the SSC nucleus and a response is created. This phase was called the physiological phase [13]. The next phase is the tactical phase, in which as a result of adverse environmental changes, SSCs form cells of different phenotype, i.e. different shapes, different metabolism, etc. At this stage, mainly morphological atrophic and hypertrophic changes are observed. If the tactical phase 
is not enough to protect the resulting daughter cells, the SSC genome jumps to the brink of chaos. This phase is called the chaos edge phase or the atavistic phase. This phase is pathomorphologically described as dysplasia or preneoplastic metaplasia, and phenotypes are referred to as atavistic phenotypes. If the SSC and daughter cells cannot adapt to an environment with increased energy flows, the systems go into the chaos or rescue phase (innovation phase). In this phase, SSCs become cancer stem cells. Delay in input signal transduction is a prerequisite for chaotic behavior $[14,15]$ These changes are „strategic" (fundamental changes in the current function of the cell). SSCs explore their own genotype spaces through, chaotic search'. Stochasticity and chaos are not identical. Chaotic sequences can be produced using deterministic algorithms [16].

The difference is important because differences in determined chaos are limited by the attractor, while true stochasticity is not limited. All chaotic innovations have their source in some genotype space. These innovations include molecules with new structures and biochemical functions. Chaos can act as a "heterogeneity engine" that allows cell populations to quickly study many phenotypes (different morphology, nuclear structure, chromatin architecture, metabolism, transmembrane potentials, etc.) [13]. Genomic chaos also refers to an increased rate of genome restructuring: changes in the number of chromosomes (aneuploidy), segmental rearrangement of chromosomes (translocations, duplications, inversions and deletions), instability of repetitive sequences and individual catastrophic events, e.g. the phenomenon of chromothripsis [14, 15]. Chromothripsis is a subtype of chaotic genome. It makes up roughly less than $10 \%$ of all different types of chaotic genomes examined [17]. The lack of experimental evidence of generating chaos at the intracellular level in vivo may indicate that during evolution the cell found a solution to this problem, namely the possibility of stabilizing the system in the presence of factors generating chaos [18]. Phenotypic diversity created by genomic chaos can be beneficial in a hostile environment, it is well established in ecology and population genetics.

The fact remains that, despite the chaos in the genome, which should generally lead to cell death, some chaotic genomes acquire the ability to survive and survive. About $45 \%$ of the human genome consists of transposable elements, which is a type of unauthorized recombination that does not require the homology of the nucleotide sequence of the DNA molecules involved. Transposable elements, which belong to the group of mobile genetic elements, due to their ability to move from one place in the genome to another, can cause large changes in the structure of the genome, i.e. inversions, deletions and duplications large DNA fragments. DNA transposition is commonly found in the human genome, but usually does not include coding sequences.

Liu et al. [19] presented evidence to support chaotic theory, showing that potential chaotic motifs are visible in the expression of certain genes in cancer cell lines.

Recently, Lou and Liu, propose the integrative theory for cancer, in which are three basic elements of cancer development - genetic alterations (cancer), metabolic imbalance (host) and immunological response (host) [20]. Cancer is a complex disease involving many changes in cell physiology and metabolism. According to Warburg the primary cause of cancer is a metabolic switch from oxidative to glycolytic metabolism. Damage the respiration and energy metabolism precedes and underlies the genome instability that accompanies tumor development [21]. According to Seyfried cancer is a mitochondrial metabolic disease [22]. The dysregulation of mitochondrial function is one the main component of the metabolic reprograming of cell. Abnormalities in the content and composition of mitochondria have been observed in different tumor tissues. The changes of metabolism can also trigger transcriptional programs alterations associated with the inflammatory milieu, acceleration cellular proliferation and metastasis [23].

The latest theory is the systemic-evolution theory of cancer (SETOC) which postulates two connected concepts. Breaking down the endosymbiosis between mitochondria and the information nucleus/cytoplasm. The evolution of the system of various cellular components because of long-term injuries leads to atavistic biological functions, dysplasia and then cancer [24]. Several studies show that destroying the communication between the mitochondria and nucleus makes the first dysfunctional and triggers carcinogenesis [25-27].

Epidemiological studies show that some of the cancers are caused by infection with a virus containing RNA-type genetic material (hepatitis c-HCV virus, retroviruses, e.g. HTLV-1) or DNA-type viruses [i.e. hepatitis B virus (HBV), Kaposi sarcoma herpesvirus (KSHV/HHV8, Merkel cell polyomavirus (MCV), Epstein-Barr virus (EBV)]. Cancer develops most often as a result of long-term infection. In addition, non-infectious cofactors, including age, genetics, environmental play an important role in the etiology of these cancers. Other factors and immunity e.g. dietary aflatoxin for HBV related hepatocellular carcinoma or host mutation predispose such as in genes encoding EVER1 and EVER2 for beta HPV-related epidermodysplasia verruciformis [28]. Individual human tumor viruses exert their malignant effects in different ways.

\section{SUMMARY}

In modern science, the existence of competing theories that differ in basic assumptions, but are based partly on 
the same experimental data, is not unusual. The solution to a certain problem, in this case theories regarding the formation of tumors, can be analyzed by the thesis that it is impossible to specify or explain the theory through given experiments. The existence of significant theoretical differences between the processes presented above results from the huge amount of experimental data and the lack of unambiguous results confirming or negating the assumptions of the researchers. It is possible that soon, thanks to advances in molecular research on carcinogenesis, the problem of the formation and treatment of cancerous cell transformation will be partially solved [29].

\section{Conflicts of interest}

The authors declare no conflict of interest.

\section{REFERENCES}

1. Sonnenschein C, Soto AM. Theories of carcinogenesis: an emerging perspective. Semin Cancer Biol. 2008; 18(5): 372-377, doi: 10.1016/j. semcancer.2008.03.012, indexed in Pubmed: 18472276.

2. Vaux D. In defense of the somatic mutation theory of cancer. BioEssays. 2011; 33(5): 341-343, doi: 10.1002/bies.201100022.

3. Hanahan D, Weinberg RA. Hallmarks of cancer: the next generation. Cell. 2011; 144(5): 646-674, doi: 10.1016/j.cell.2011.02.013, indexed in Pubmed: 21376230.

4. Ye CJ, Sharpe Z, Alemara S, et al. Micronuclei and Genome Chaos: Changing the System Inheritance. Genes (Basel). 2019; 10(5), doi: 10.3390/genes10050366, indexed in Pubmed: 31086101.

5. Duesberg P, Li R, Fabarius A, et al. The Chromosomal Basis of Cancer. Anal Cell Pathol. 2005; 27(5-6): 293-318, doi: 10.1155/2005/951598.

6. Baker SG, Vaux DL, Soto AM, et al. The tissue organization field theory of cancer: a testable replacement for the somatic mutation theory. Bioessays. 2011; 33(5): 332-340, doi: 10.1002/bies.201100025, indexed in Pubmed: 21503935.

7. Vong $S$, Kalluri R. The role of stromal myofibroblast and extracellular matrix in tumor angiogenesis. Genes Cancer. 2011; 2(12): 1139-1145, doi: 10.1177/1947601911423940, indexed in Pubmed: 22866205.

8. Li JW, Yang D, Yang D, et al. Tumors arise from the excessive repair of damaged stem cells. Med Hypotheses. 2017; 102: 112-122, doi: 10.1016/j. mehy.2017.03.005, indexed in Pubmed: 28478815.

9. Bedessem B, Ruphy S, Bedessem B, et al. SMT or TOFT? How the two main theories of carcinogenesis are made (artificially) incompatible. Acta Biotheor. 2015; 63(3): 257-267, doi: 10.1007/s10441-015-9252-1, indexed in Pubmed: 25851566.

10. Tomasetti C, Vogelstein B. Cancer etiology. Variation in cancer risk among tissues can be explained by the number of stem cell divisions. Science. 2015; 347(6217): 78-81, doi: 10.1126/science.1260825, indexed in Pubmed: 25554788

11. Mangel $M$, Bonsall MB. Phenotypic evolutionary models in stem cell biology: replacement, quiescence, and variability. PLoS One. 2008; 3(2): e1591, doi: 10.1371/journal.pone.0001591, indexed in Pubmed: 18270578.
12. Braun $E$. The unforeseen challenge: from genotype-to-phenotype in cell populations. Rep Prog Phys. 2015; 78(3): 036602, doi: 10.1088/00344885/78/3/036602, indexed in Pubmed: 25719211.

13. Suzuki Y, Lu M, Ben-Jacob E, et al. Periodic, Quasi-periodic and Chaotic Dynamics in Simple Gene Elements with Time Delays. Sci Rep. 2016; 6: 21037, doi: 10.1038/srep21037, indexed in Pubmed: 26876008.

14. Agur Z, Kogan Y, Levi L, et al. Disruption of a Quorum Sensing mechanism triggers tumorigenesis: a simple discrete model corroborated by experiments in mammary cancer stem cells. Biol Direct. 2010; 5: 20, doi: 10.1186/1745-6150-5-20, indexed in Pubmed: 20406437.

15. Weiss H. A Mathematical Introduction to Population Dynamics. IMPA Publicacões Matemáticas 2009.

16. Tez M, Tez S. Chaos, Adaptation and Information are the Main Pillars of Carcinogenesis. , doi: 10.20944/preprints201906.0256.v1.

17. Heng HHQ, Liu G, Stevens JB, et al. Karyotype heterogeneity and unclassified chromosomal abnormalities. Cytogenet Genome Res. 2013; 139(3): 144-157, doi: 10.1159/000348682, indexed in Pubmed: 23571381.

18. Khlebodarova TM, Kogai VV, Fadeev Sl, et al. Chaos and hyperchaos in simple gene network with negative feedback and time delays. J Bioinform Comput Biol. 2017; 15(2): 1650042, doi: 10.1142/S0219720016500426, indexed in Pubmed: 28052708.

19. Liu D, Albergante $L$, Newman TJ. Universal attenuators and their interactions with feedback loops in gene regulatory networks. Nucleic Acids Res. 2017; 45(12): 7078-7093, doi: 10.1093/nar/gkx485, indexed in Pubmed: 28575450.

20. Luo G, Liu Na. An integrative theory for cancer (Review). Int J Mol Med 2018, doi: 10.3892/ijmm.2018.4004.

21. Poljsak B, Kovac V, Dahmane R, et al. Cancer Etiology: A Metabolic Disease Originating from Life's Major Evolutionary Transition? Oxid Med Cell Longev. 2019; 2019: 7831952, doi: 10.1155/2019/7831952, indexed in Pubmed: 31687086.

22. Seyfried TN. Cancer as a mitochondrial metabolic disease. Front Cell Dev Biol. 2015; 3: 43, doi: 10.3389/fcell.2015.00043, indexed in Pubmed: 26217661.

23. Neagu M, Constantin C, Popescu ID, et al. Inflammation and Metabolism in Cancer Cell-Mitochondria Key Player. Front Oncol. 2019; 9: 348, doi: 10.3389/fonc.2019.00348, indexed in Pubmed: 31139559.

24. Mazzocca A. The Systemic-Evolutionary Theory of the Origin of Cancer (SETOC): A New Interpretative Model of Cancer as a Complex Biological System. Int J Mol Sci. 2019; 20(19), doi: 10.3390/ijms20194885, indexed in Pubmed: 31581628.

25. Seyfried TN, Flores RE, Poff AM, et al. Cancer as a metabolic disease: implications for novel therapeutics. Carcinogenesis. 2014; 35(3): 515-527, doi: 10.1093/carcin/bgt480, indexed in Pubmed: 24343361.

26. Xia M, Zhang Y, Jin Ke, et al. Communication between mitochondria and other organelles: a brand-new perspective on mitochondria in cancer. Cell Biosci. 2019; 9: 27, doi: 10.1186/s13578-019-0289-8, indexed in Pubmed: 30931098.

27. Mello T, Simeone I, Galli A. Mito-Nuclear Communication in Hepatocellular Carcinoma Metabolic Rewiring. Cells. 2019; 8(5), doi: 10.3390/cells8050417, indexed in Pubmed: 31060333.

28. Tommasino M. The biology of beta human papillomaviruses. Virus Res. 2017; 231: 128-138, doi: 10.1016/j.virusres.2016.11.013, indexed in Pubmed: 27856220.

29. Mazzocca A, Ferraro G, Misciagna G, et al. Moving the systemic evolutionary approach to cancer forward: Therapeutic implications. Med Hypotheses. 2018;121:80-87, doi: 10.1016/j.mehy.2018.09.033, indexed in Pubmed: 30396500. 